\title{
Metformin and pioglitazone combination therapy ameliorate polycystic ovary syndrome through AMPK/PI3K/JNK pathway
}

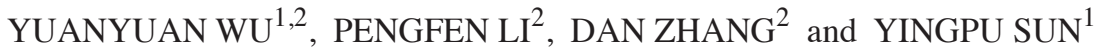 \\ ${ }^{1}$ Reproductive Medicine Center, The First Affiliated Hospital of Zhengzhou University, Zhengzhou, Henan 450052; \\ ${ }^{2}$ Reproductive Medicine Center, The Second Affiliated Hospital of Zhengzhou University, \\ Zhengzhou, Henan 450014, P.R. China
}

Received February 19, 2016; Accepted February 7, 2017

DOI: $10.3892 / \mathrm{etm} .2017 .5650$

\begin{abstract}
Polycystic ovary syndrome (PCOS) is a common gynecological endocrine disorder, which results in health problems such as menstrual disorders, hyperandrogenism and persistent anovulation. Hyperandrogenism and insulin resistance are the basic characteristics of PCOS. To investigate the combined effect of metformin and pioglitazone on POCS and the potential mechanisms, a rat model of PCOS was established by intramuscular injection of estradiol valerate (EV). The effect of metformin and pioglitazone monotherapy or combination therapy in control rats and PCOS rats was evaluated, involving the testosterone level, follicular development and insulin resistance. The potential mechanism for the therapeutic effect of metformin and pioglitazone on POCS was explored through using three inhibitors of the 5'adenosine monophosphate-activated protein kinase (AMPK)/phosphoinositide-3 kinase (PI3K)/c-Jun N-terminal kinase (JNK) pathway (Compound C, Wortmannin and SP600125). The results showed that EV-induced PCOS rats demonstrated hyperandrogenemia, hyperinsulinemia and follicular dysplasia. Metformin or pioglitazone monotherapy significantly suppressed the high level of testosterone, reduced the raised percentage of cystic follicles and primary follicles, promoted the number of early antral follicles, and markedly decreased the high concentration of fasting insulin and homeostatic model assessment for insulin resistance index in PCOS rats. In addition, metformin and pioglitazone combination therapy demonstrated greater efficacy than its individual components. Furthermore, individual or joint treatment with metformin and pioglitazone affected the phosphorylation level of JNK in PCOS rats. Compound C and Wortmannin eliminated the effect of metformin and
\end{abstract}

Correspondence to: Dr Yingpu Sun, Reproductive Medicine Center, The First Affiliated Hospital of Zhengzhou University, 1 Jianshe East Road, Zhengzhou, Henan 450052, P.R. China

E-mail: syp_rmc@163.com

Key words: metformin, pioglitazone, polycystic ovary syndrome, AMPK/PI3K/JNK pathway pioglitazone combination therapy on improving the follicular growth in PCOS rats, whereas SP600125 treatment enhanced this combination therapy effect. These data suggested that metformin and pioglitazone combination therapy demonstrated great efficacy in ameliorating PCOS through regulating the AMPK/PI3K/JNK pathway.

\section{Introduction}

Polycystic ovary syndrome (PCOS) is a common gynecological endocrine disorder that affects $5-10 \%$ of reproductive-age women $(1,2)$. It is a complex and intractable disorder with psychological (increased anxiety, depression and worsened quality of life), reproductive (infertility, hyperandrogenism and hirsutism) and metabolic manifestations [insulin resistance, impaired glucose tolerance, type 2 diabetes mellitus (T2DM) and adverse cardiovascular risk profiles] (3). Due to menstrual disorders, hyperandrogenism and persistent anovulation, PCOS is considered to be the main cause of infertility in women (4). An estimate of 50-70\% of women with PCOS have insulin resistance (5). Insulin resistance has a critical role in the pathogenesis of PCOS (6). In addition to induced hyperandrogenism and other clinical characteristics of PCOS, insulin resistance is also involved in cardiovascular diseases and diabetes mellitus in PCOS patients (7,8). At present,insulin resistance and reproductive dysfunction (hyperandrogenism and anovulation) do not receive sufficient attention during the treatment of PCOS.

Clomifene citrate, metformin and thiazolidinediones (TZDs, e.g., rosiglitazone and pioglitazone), which are often used to improve insulin sensitivity and reduce insulin resistance, are considered the first-line ovulation-inducing drugs in infertile women with PCOS either alone or in combination (9-14). Metformin, an adenosine monophosphate-activated protein kinase (AMPK) activator and anti-diabetic agent of the biguanide class, is widely used for the treatment of T2DM (15-18). In PCOS patients, it has been confirmed that metformin treatment decreases hyperandrogenemia, reestablishes the menstrual cycle, affects the endometrial thickness and blood flow, and ultimately improves ovulation, implantation and pregnancy rates $(19,20)$. TZDs are the peroxisome proliferator-activated receptor $\gamma$ synthetic ligands that regulate cellular functions to decrease insulin resistance $(21,22)$. 
In a previous study, pioglitazone showed an obvious beneficial effect on insulin sensitivity in patients with impaired fasting glucose and glucose tolerance $(23,24)$. In women with PCOS, pioglitazone treatment was found to improve the irregularities of menses and hirsutism, reduce insulin resistance, increase the ovulation rate and improve hyperandrogenemia (25-28). Pioglitazone and metformin fixed-dose combination therapy was demonstrated to be well tolerated, with reduced or similar adverse event rates in treating patients with T2DM compared with each individual monotherapy (29). In addition, it was found that combined treatment with letrozole, metformin and pioglitazone was efficacious among women with PCOS resistant to clomifene citrate, and it was similar to that of combined treatment with clomifene citrate, metformin and pioglitazone (14). However, the exact efficacy and potential mechanism for the combination therapy of metformin and pioglitazone in PCOS has remained to be elucidated.

Based on this, the aim of the present study was to investigate the combined effect of metformin and pioglitazone on the testosterone levels, follicular development and insulin resistance in a rat model of PCOS, and to explore the potential mechanism through using three inhibitors of the AMPK/phosphoinositide-3 kinase $(\mathrm{PI} 3 \mathrm{~K})$ /c-Jun N-terminal kinase (JNK) pathway (Compound C, Wortmannin and SP600125).

\section{Materials and methods}

Animals and drugs. Female Sprague-Dawley rats (age, 3 weeks; n=168; weight, 50-55g) were obtained from JRDUN Biotechnology (Shanghai, China) and housed in a pathogen-free environment at the animal center of the First Affiliated Hospital of Zhengzhou University (Zhengzhou, China) maintained under standard conditions (room temperature, $22^{\circ} \mathrm{C}$; humidity, $33 \%$ ) with a 12 -h light/dark cycle. All experimental operations were performed in accordance with the Guide for the Care and Use of Laboratory Animals (30). Metformin and pioglitazone were purchased from Sigma-Aldrich (Merck KGaA, Darmstadt, Germany). Compound C as an AMPK inhibitor was purchased from Calbiochem (San Diego, CA, USA). Wortmannin as a PI3K inhibitor and SP600125 as a JNK inhibitor were purchased from Sigma-Aldrich. The study protocol was approved by the Medical Ethics Committee of the First Affiliated Hospital of Zhengzhou University and the Second Affiliated Hospital of Zhengzhou University (Zhengzhou, China).

PCOS rat model. The PCOS rat model was induced by a single dose of estradiol valerate ( $\mathrm{EV} ; 4 \mathrm{mg} / \mathrm{rat}$, intramuscular) as described previously (31). The rats were divided into eight groups ( $\mathrm{n}=12$ per group) as follows: Control group (Control Veh), normal rats treated with $500 \mathrm{mg} / \mathrm{kg} /$ day metformin orally (Control Met), normal rats treated with $20 \mathrm{mg} / \mathrm{kg} /$ day pioglitazone orally (Control Pio), normal rats treated with $500 \mathrm{mg} / \mathrm{kg} /$ day metformin plus $20 \mathrm{mg} / \mathrm{kg} /$ day pioglitazone orally (Control Met + Pio), PCOS group (PCOS Veh), PCOS rats treated with $500 \mathrm{mg} / \mathrm{kg} /$ day metformin orally (PCOS Met), PCOS rats treated with $20 \mathrm{mg} / \mathrm{kg} /$ day pioglitazone orally (PCOS Pio) and PCOS rats treated with $500 \mathrm{mg} / \mathrm{kg} /$ day metformin plus $20 \mathrm{mg} / \mathrm{kg} /$ day pioglitazone orally (PCOS Met + Pio). At the end of the treatment, blood samples and ovaries were collected for the biochemical and histological evaluations.

$A M P K / P I 3 K / J N K$ pathway inhibitor treatment. To explore the potential mechanism of the therapeutic effect of metformin and pioglitazone on PCOS, compound C, Wortmannin and SP600125 were used to inhibit the AMPK/PI3K/JNK pathway. The rats were divided into six groups ( $n=12$ per group) as follows: Control group (Control), PCOS group (PCOS), PCOS rats treated with $500 \mathrm{mg} / \mathrm{kg} /$ day metformin plus $20 \mathrm{mg} / \mathrm{kg} /$ day pioglitazone orally (PCOS + Met + Pio), PCOS rats co-treated with $500 \mathrm{mg} / \mathrm{kg} /$ day metformin, $20 \mathrm{mg} / \mathrm{kg} /$ day pioglitazone orally and Compound C [25 $\mu \mathrm{g} / \mathrm{rat}$, intravenously (i.v.)] $(\mathrm{PCOS}+\mathrm{Met}+\mathrm{Pio}+\mathrm{CC}), \mathrm{PCOS}$ rats co-treated with $500 \mathrm{mg} / \mathrm{kg} /$ day metformin, $20 \mathrm{mg} / \mathrm{kg} /$ day pioglitazone orally and wortmannin $(15 \mu \mathrm{g} / \mathrm{kg}$, i.v. $)(\mathrm{PCOS}+\mathrm{Met}+\mathrm{Pio}+\mathrm{WT})$, and PCOS rats co-treated with $500 \mathrm{mg} / \mathrm{kg} /$ day metformin, $20 \mathrm{mg} / \mathrm{kg} /$ day pioglitazone orally and SP600125 $(0.05 \mathrm{mg} / \mathrm{kg}$, i.v. $(\mathrm{PCOS}+\mathrm{Met}+\mathrm{Pio}+\mathrm{SP})$. At the end of the treatment, the ovaries were collected for the histological evaluations.

Assessment of biochemical parameters. At the end of the treatment, the rats were fasted overnight and blood samples were collected. Blood was centrifuged at 15,500 x g for $5 \mathrm{~min}$, and the plasma and serum samples were separated for determination of the biochemical parameters. Fasting glucose concentrations were determined using the glucose oxidase method as described previously (32). ELISA was used to assess free testosterone, total testosterone and fasting insulin according to the manufacturer's instructions. The kits were purchased from Sigma-Aldrich. The homeostasis model assessment of insulin resistance index (HOMA-IR) was calculated as plasma glucose $(\mathrm{mmol} / \mathrm{l}) \mathrm{x}$ serum insulin $(\mathrm{mIU} / \mathrm{l}) / 22.5$.

Assessment of histological changes. The ovary samples were removed from each group and immediately fixed in Bouin solution for $12 \mathrm{~h}$ and then embedded in paraffin. Sections (5 $\mu \mathrm{m}$ thickness) were mounted on the glass slides and stained with hematoxylin-eosin (H\&E) for histological examination under the microscope. The different structures per ovary section were counted. Follicles were classified as cystic follicles, primary follicles, early antral follicles and atretic follicles according to the description by Di Pietro et al (33).

Western blot analysis. Western blot analysis was performed as described previously (33). In brief, proteins extracted from ovary tissues were evaluated by the Bradford assay and separated by $10 \%$ SDS-PAGE (30 $\mu \mathrm{g} /$ lane), then transferred to polyvinylidene difluoride membranes (Merck KGaA, Darmstadt, Germany). Membranes were incubated with primary antibodies at $4^{\circ} \mathrm{C}$ overnight followed by processing with horseradish peroxidase-conjugated secondary antibodies at room temperature for $2 \mathrm{~h}$ and detected by chemiluminescence (SC-2048; Santa Cruz Biotechnology, Inc., Dallas, TX, USA). $\beta$-actin was used as protein loading control. Primary antibodies p-JNK (sc-6254; 1:500), JNK (sc-7345; 1:500), $\beta$-actin (sc-58673; 1:1,000) and the corresponding secondary antibodies (sc-516102; 1:2,000) were purchased from Santa Cruz Biotechnology, Inc. 
A

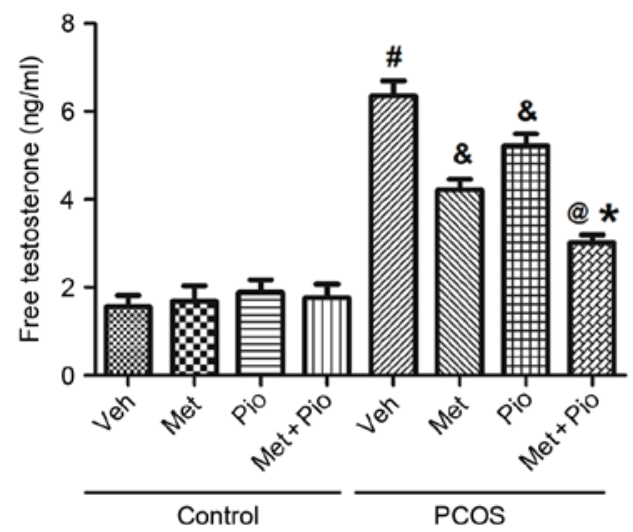

B

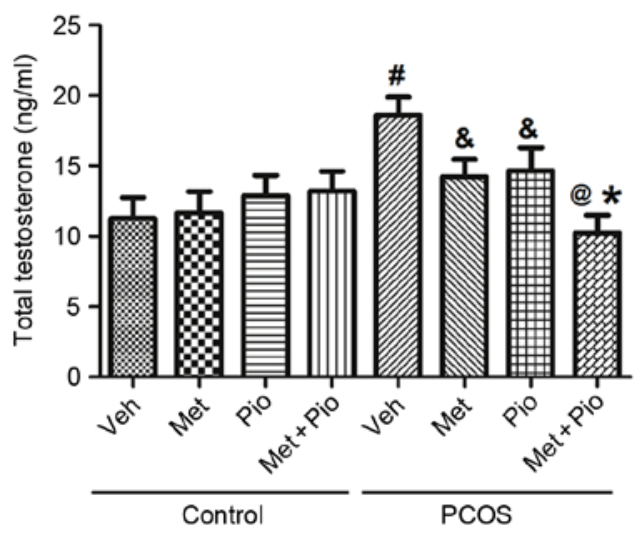

Figure 1. Level of testosterone in control and PCOS rats after individual or combined treatment with Met and Pio. The testosterone concentration in the serum was measured by ELISA. (A) Level of free testosterone. (B) Level of total testosterone. "P<0.05 vs. Veh in Control; ${ }^{\mathrm{P}}<0.05$ compared to Veh in PCOS; ${ }^{\circledR} \mathrm{P}<0.05$ vs. Met in PCOS; ${ }^{*} \mathrm{P}<0.05$ vs. Pio in PCOS. Veh, vehicle; PCOS, polycystic ovary syndrome; Pio, pioglitazone; Met, metformin.

Statistical analysis. Values are expressed as the mean \pm standard deviation. The significance of differences was determined using one-way factorial analysis of variance. Statistical analysis was performed using SPSS software version 16.0 (SPSS, Inc., Chicago, IL, USA). $\mathrm{P}<0.05$ was considered to indicate a statistically significant difference.

\section{Results}

Metformin, pioglitazole and their combination inhibit increases in testosterone levels in a PCOS model. Hyperandrogenism is the central feature of PCOS. In order to analyze the effect of metformin and pioglitazone on PCOS, the testosterone level was first determined in all groups. In the control rats, the hormone levels of free testosterone and total testosterone were not affected by metformin and pioglitazone treatment alone or combination (Fig. 1A and B). However, in PCOS rats, a significant increase was observed in the testosterone level, compared to that in the Control Veh group (free testosterone $6.35 \pm 0.35$ vs. $1.56 \pm 0.26$; total testosterone, $18.59 \pm 1.29$ vs. $11.25 \pm 1.52 ; \mathrm{P}<0.05)$. In addition, compared to that in the PCOS Veh group, metformin or pioglitazone treatment of PCOS rats significantly inhibited the level of free testosterone (PCOS Met, 4.22 \pm 0.24 ; PCOS Pio, 5.21 $\pm 0.28 ; \mathrm{P}<0.05$ ) and total testosterone (PCOS Met, 14.23 \pm 1.24 ; PCOS Pio, 14.66 \pm 1.64 ; $\mathrm{P}<0.05)$. Furthermore, the inhibition of testosterone levels was intensified with combined treatment of metformin and pioglitazone, compared to that with monotreatment (free testosterone, $3.01 \pm 0.18$; total testosterone, $10.23 \pm 1.25 ; \mathrm{P}<0.05$ ). These results suggested that metformin and pioglitazone combination therapy reduced the elevated testosterone levels in PCOS.

Metformin and pioglitazone improve ovary follicular development in PCOS. The endocrine disorder in PCOS is characterized by chronic anovulation and high androgen levels. Therefore, the present study examined the development of four types of follicles (cystic follicles, primary follicles, early antral follicles and atretic follicles) in $\mathrm{H} \& \mathrm{E}$-stained ovary histological sections. In control rats, no differences were found in the percentage of cystic follicles, primary follicles, early antral follicles and atretic follicles between the Control Veh group and the drug-treated groups (Fig. 2). By contrast, it was found that the percentage of cystic follicles in the PCOS Veh group was higher than that in the Control Veh group $(7.58 \pm 1.25$ vs. $0.50 \pm 0.25 ; \mathrm{P}<0.05)$ (Fig. 2A). When PCOS rats were treated with metformin or pioglitazone, the percentage of cystic follicles was significantly reduced compared to that in the PCOS Veh group (PCOS Met, 4.21 \pm 1.11 ; PCOS Pio, 5.22 \pm 1.22 ; $\mathrm{P}<0.05$ ). In addition, co-treatment with metformin and pioglitazone in PCOS rats promoted the inhibitory effect of metformin or pioglitazone treatment alone on the folliculogenesis of cystic follicles $(3.10 \pm 0.58 ; \mathrm{P}<0.05)$. Similar to the cystic follicles, the percentage of primary follicles in the PCOS Veh group was obviously increased compared to that in the Control Veh group $(15.68 \pm 2.22$ vs. $8.55 \pm 1.22 ; \mathrm{P}<0.05)$ (Fig. 2B). Treatment with pioglitazone decreased the folliculogenesis of primary follicles, which was comparable with the effect of metformin; furthermore, pioglitazone enhanced the ability of metformin to inhibit primary follicles in PCOS rats (PCOS Met, 12.22 \pm 1.15 ; PCOS Pio, 11.25 \pm 1.26 ; PCOS Met + Pio, $7.55 \pm 1.14 ; \mathrm{P}<0.05)$. In addition, the percentage of early antral follicles in PCOS rats was not changed as compared to that in the control rats (Fig. 2C). However, metformin or pioglitazone monotreatment markedly increased the percentage of early antral follicles in PCOS rats compared to that in the PCOS Veh group, while combined metformin with pioglitazone in PCOS rats increased this impact on the folliculogenesis of early antral follicles (PCOS Met, 59.36 \pm 3.26 ; PCOS

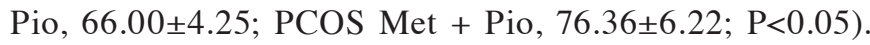
Regarding atretic follicles, their percentage was not different between all of the Control and PCOS groups except for the PCOS Met + Pio group (Fig. 2D). Compared with monotreatment, combined metformin with pioglitazone significantly suppressed the percentage of atretic follicles in PCOS rats (PCOS Met, 42.22 \pm 5.70 ; PCOS Pio, 41.25 \pm 5.23 ; PCOS Met + Pio, 27.55 $\pm 4.14 ; \mathrm{P}<0.05)$. Taken together, these results implied that treatment with metformin and pioglitazone improved ovary follicular development in PCOS.

Metformin and pioglitazone reduce elevated insulin levels and ameliorate insulin resistance in a PCOS model. Insulin 
A

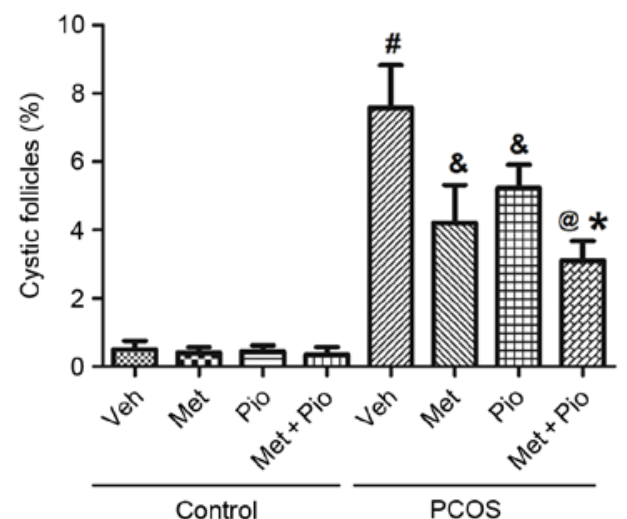

C

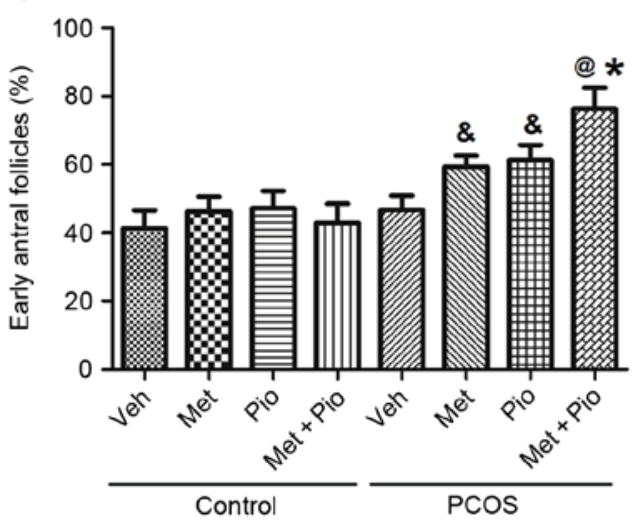

B

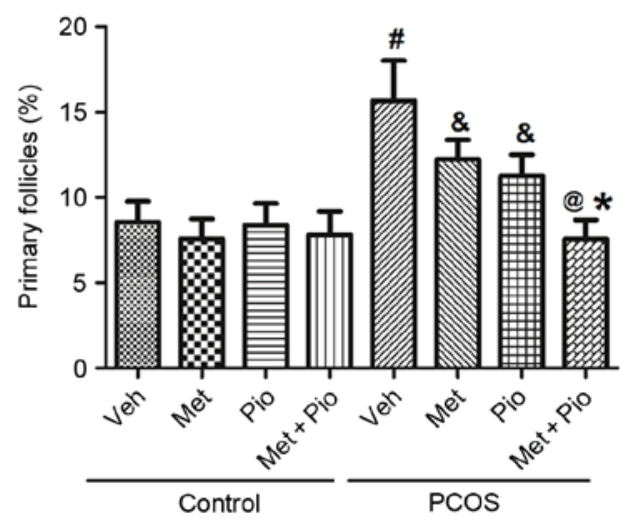

D

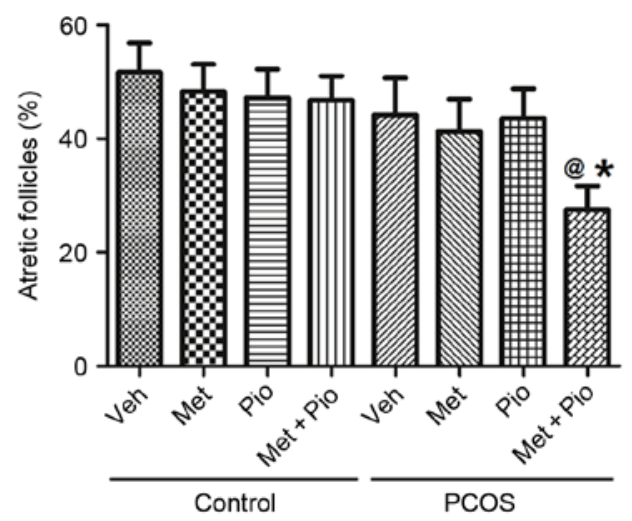

Figure 2. Follicular development in control and PCOS rats after individual or combined treatment with Met and Pio. The graphs show the percentage of four types of follicle in ovarian sections stained with hematoxylin and eosin. The percentage of (A) cystic follicles, (B) primary follicles, (C) early antral follicles and (D) atretic follicles. ${ }^{*} \mathrm{P}<0.05$ compared to Veh in Control; ${ }^{\circledR} \mathrm{P}<0.05$ compared to Veh in PCOS; ${ }^{\circledR} \mathrm{P}<0.05$ compared to Met in PCOS; $\mathrm{P}<0.05$ compared to Pio in PCOS. Veh, vehicle; PCOS, polycystic ovary syndrome; Pio, pioglitazone; Met, metformin.
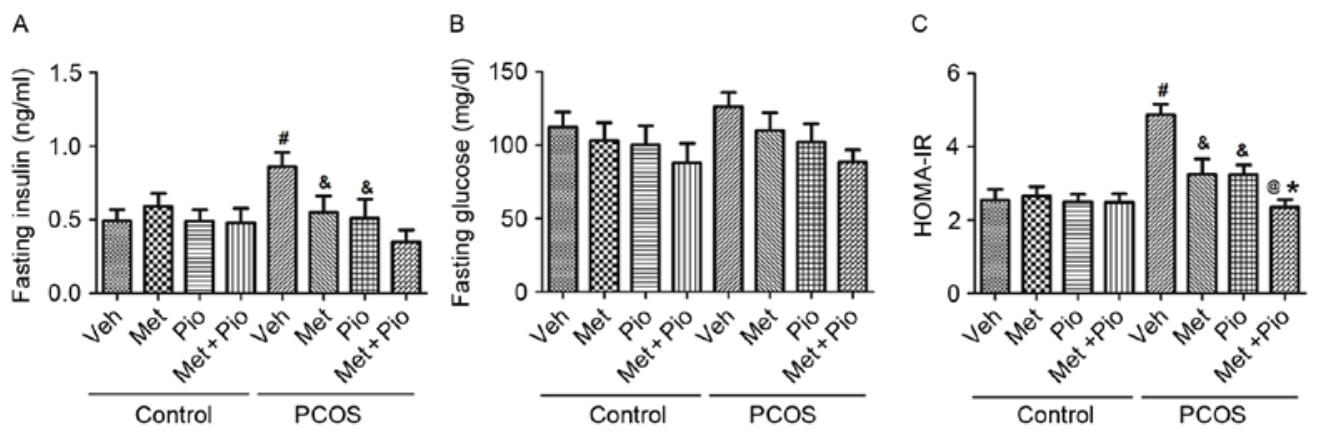

Figure 3. Level of fasting insulin and glucose in control and PCOS rats after individual or combined treatment with Met and Pio. (A) The concentration of fasting insulin in the serum measured by ELISA. (B) The concentration of fasting glucose measured using the glucose oxidase method. (C) HOMA-IR calculated as plasma glucose (mmol/l) x serum insulin $(\mathrm{mIU} / \mathrm{l}) / 22.5 .{ }^{*} \mathrm{P}<0.05$ compared to Veh in Control; ${ }^{\text {* }} \mathrm{P}<0.05$ compared to Veh in $\mathrm{PCOS} ;{ }^{\circledR} \mathrm{P}<0.05$ compared to Met in PCOS; ${ }^{*} \mathrm{P}<0.05$ compared to Pio in PCOS. Veh, vehicle; PCOS, polycystic ovary syndrome; Pio, pioglitazone; Met, metformin; HOMA-IR, homeostasis model assessment of insulin resistance.

resistance/hyperinsulinemia is the basic characteristic of metabolic abnormalities in patients with PCOS. As expected, in PCOS rats, the fasting insulin was higher than that in the control rats $(0.86 \pm 0.14$ vs. $0.55 \pm 0.12 ; \mathrm{P}<0.05)$ (Fig. $3 \mathrm{~A})$, Fasting glucose levels did not significantly change in PCOS rats compared with control rats (Fig. 3B). HOMA-IR increased in PCOS rats compared with control rats $(4.88 \pm 0.28$ vs. $2.55 \pm 0.29$; $\mathrm{P}<0.05)$, suggesting insulin resistance was present in PCOS rats (Fig. 3C). Metformin treatment in PCOS rats significantly inhibited the increase in the insulin concentration and the

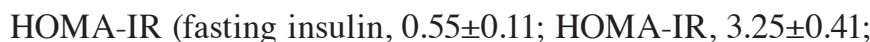
$\mathrm{P}<0.05)$. Pioglitazone treatment showed a similar effect on PCOS rats to that of metformin (fasting insulin, 0.51 \pm 0.13 ; HOMA-IR, 3.24 \pm 0.26 ; $\mathrm{P}<0.05)$. Furthermore, combined metformin with pioglitazone significantly enhanced the inhibitory effect of the respective monotreatments on the insulin concentration and the HOMA-IR in PCOS rats (fasting insulin, 0.35 \pm 0.08 ; HOMA-IR, 2.35 \pm 0.21 ; $\mathrm{P}<0.05$ ). However, in the control rats, the levels of fasting insulin, fasting glucose and HOMA-IR were not affected by metformin and 

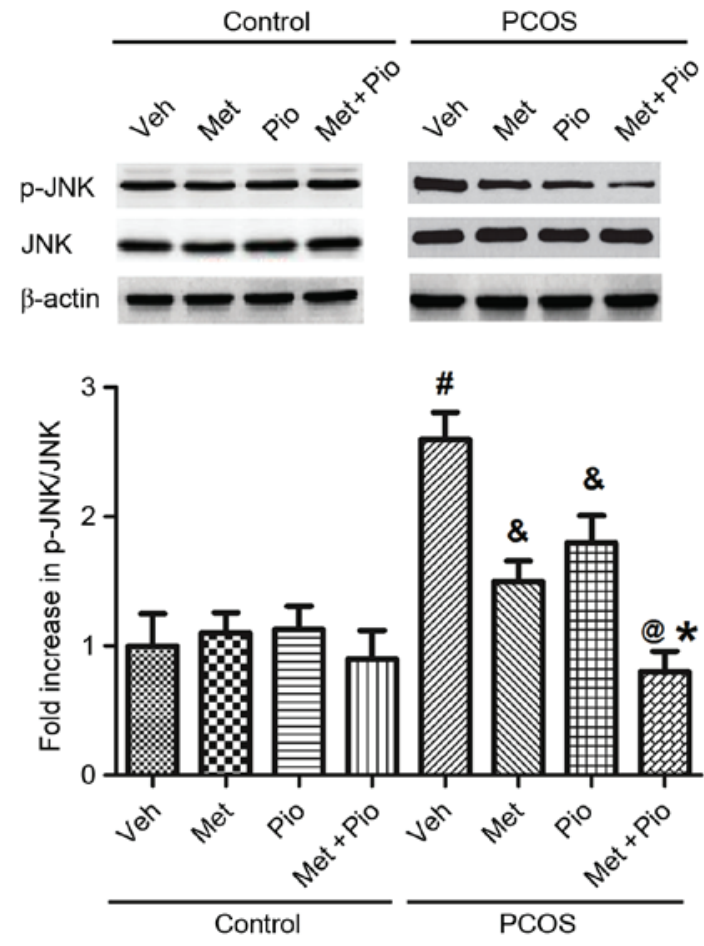

Figure 4. Phosphorylation of JNK in control and PCOS rats after individual or combined treatment with Met and Pio. The relative levels of phosphorylated JNK in ovarian protein extracts were determined by western blot analysis. The density in each band was normalized to that of the $\beta$-actin band. ${ }^{*} \mathrm{P}<0.05$ compared to Veh in Control; ${ }^{\&} \mathrm{P}<0.05$ compared to Veh in PCOS; ${ }^{\circ} \mathrm{P}<0.05$ compared to Met in PCOS; " $\mathrm{P}<0.05$ compared to Pio in PCOS. p-JNK, phosphorylated c-Jun N-terminal kinase; Veh, vehicle; PCOS, polycystic ovary syndrome; Pio, pioglitazone; Met, metformin.

pioglitazone compared to those in the Control Veh group. The results showed that metformin and pioglitazone combination therapy reduced the high levels of insulin and ameliorated insulin resistance in PCOS.

Metformin and pioglitazone mediate follicular development in PCOS through the AMPK/PI3K/JNK pathway. A previous study showed that metformin mediated thapsigargin-induced apoptosis and insulin secretion impairment via regulation of the AMPK/PI3K/JNK pathway (34). Therefore, the present study sought to explore whether the effect of metformin and pioglitazone on PCOS was mediated through the AMPK/PI3K/JNK pathway. First, the phosphorylation of JNK after metformin and pioglitazone treatment was detected. As shown in Fig. 4, JNK phosphorylation was significantly induced in PCOS rats, which was suppressed by metformin or pioglitazone treatment $(\mathrm{P}<0.05)$. Co-treatment with metformin and pioglitazone aggravated the inhibition of JNK phosphorylation in PCOS rats $(\mathrm{P}<0.05$; Fig. 4). However, individual or combined treatment with metformin and pioglitazone did not affect the phosphorylation level of JNK in control rats.

Next, the involvement of the AMPK/PI3K/JNK pathway in metformin- and pioglitazone-induced improvement of the follicular development in PCOS was examined by using compound C (an AMPK inhibitor), Wortmannin (a PI3K inhibitor) and SP600125 (a JNK inhibitor). In agreement with the results shown in Fig. 2, the percentage of cystic follicles and primary follicles was significantly increased in PCOS rats, and the folliculogenesis of cystic follicles, primary follicles and atretic follicles was obviously inhibited after metformin and pioglitazone combination therapy in PCOS rats, while the percentage of early antral follicles was promoted (PCOS: Cystic follicles, 8.55 \pm 0.92 ; primary follicles, $18.57 \pm 1.36$; early antral follicles, $48.55 \pm 6.59$; atretic follicles, 49.55 \pm 6.85 ; PCOS + Met + Pio: Cystic follicles, $3.59 \pm 0.45$; primary follicles, 7.14 \pm 1.10 ; early antral follicles, 76.58 \pm 9.64 ; atretic follicles, 25.46 \pm 5.18 ) ( $\mathrm{P}<0.05$; Fig. 5). Furthermore, Compound $\mathrm{C}$ and Wortmannin eliminated the effect of metformin and pioglitazone combination therapy on the follicular development in PCOS rats (PCOS + Met + Pio + CC: Cystic follicles, 7.55 \pm 0.66 ; primary follicles $15.24 \pm 1.22$; early antral follicles, $55.69 \pm 6.78$; atretic follicles, 49.56 \pm 4.66 ; PCOS + Met + Pio + WT: Cystic follicles, $7.04 \pm 0.48$; primary follicles, $16.66 \pm 1.69$; early antral

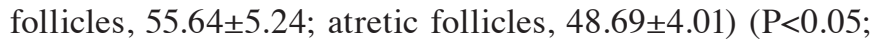
Fig. 5). Conversely, the JNK inhibitor SP600125 significantly enhanced the effect of metformin and pioglitazone-mediated follicular development in PCOS rats $(\mathrm{PCOS}+\mathrm{Met}+\mathrm{Pio}+\mathrm{SP}$ : Cystic follicles, 2.58 \pm 0.18 ; primary follicles, $4.25 \pm 0.69$; early antral follicles, $80.59 \pm 6.33$; atretic follicles, $16.44 \pm 3.25$ ) $(\mathrm{P}<0.05$; Fig. 5).

Taken together, these results suggested that metformin and pioglitazone regulate the follicular development in PCOS through the AMPK/PI3K/JNK pathway.

\section{Discussion}

PCOS is characterized by three major consequences: Hyperandrogenemia, hyperinsulinemia and abnormal ovulation. The present study evaluated the combined therapeutic effect of metformin and pioglitazone on these major symptoms of PCOS and found that metformin and pioglitazone monotherapy or combination therapy did not impact the testosterone level, insulin concentration and ovarian follicular development in control rats, but demonstrated great efficacy in inhibiting increases in testosterone levels and insulin concentration, reducing insulin resistance and ameliorating ovarian development in PCOS rats. Furthermore, individual or joint treatment with metformin and pioglitazone affected the phosphorylation levels of JNK in PCOS rats. The AMPK/PI3K/JNK pathway was demonstrated to regulate the effect of metformin and pioglitazone combination therapy on improving the follicular growth in PCOS rats.

Previous studies have evaluated the treatment with metformin and pioglitazone alone or in different combinations with other drugs for hormone modulation, ovulation induction and insulin resistance in women with PCOS (14,18,19,35-38). After metformin monotreatment, circulating testosterone, $\Delta$-4-androstenedione and the hirsutism score in PCOS patients were significantly decreased, indicating that metformin treatment led to a reduction of hyperandrogenism (39). This result was consistent with the finding of the present study that metformin treatment in PCOS rats significantly inhibited the level of free and total testosterone. In addition, the present results showed that pioglitazone and metformin have equivalent efficacy in reducing hyperandrogenism and insulin resistance in PCOS. Similar to metformin treatment, pioglitazone monotreatment significantly inhibited the increases of free testosterone, total 
A
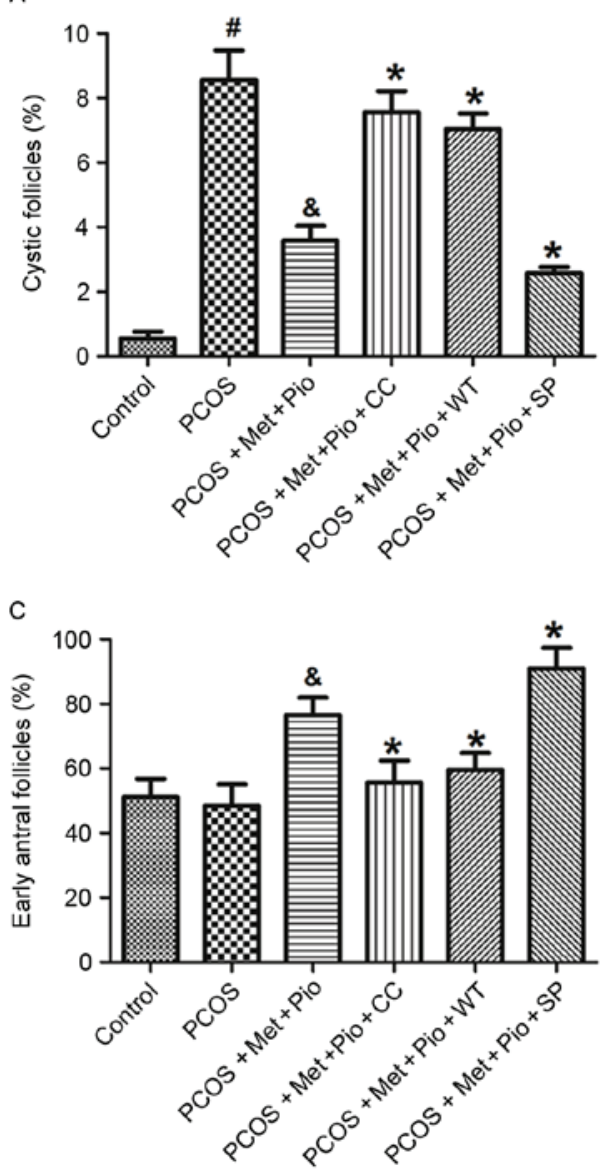

B

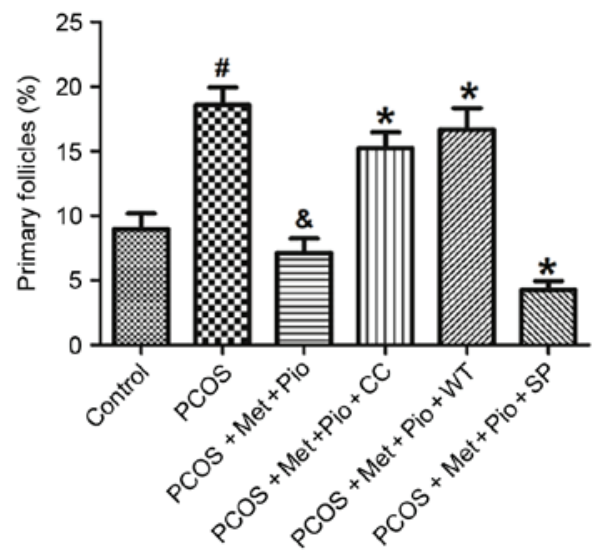

D

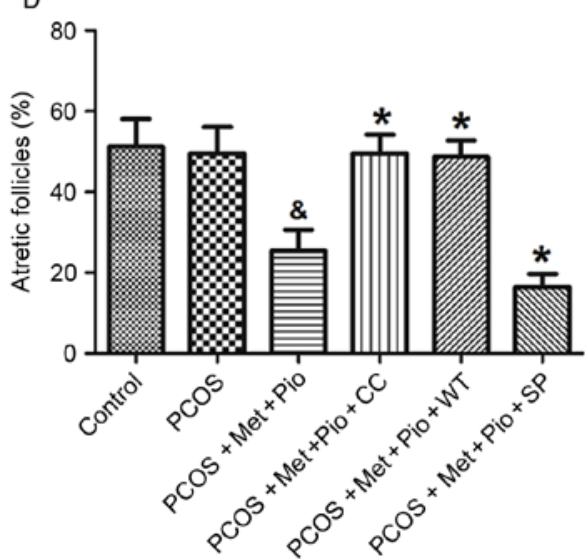

Figure 5. Follicular development in Met- and Pio-treated PCOS rats after treatment with adenosine monophosphate-activated protein kinase/phosphoinositide-3 kinase c-Jun N-terminal kinase pathway inhibitors. The graphs show the percentage of four types of follicles in ovarian sections stained with hematoxylin and eosin. The percentage of (A) cystic follicles, (B) primary follicles, (C) early antral follicles and (D) atretic follicles. ${ }^{~} \mathrm{P}<0.05$ compared to Control; ${ }^{\circ} \mathrm{P}<0.05$ compared to PCOS; "P<0.05 compared to PCOS + Met + Pio. PCOS, polycystic ovary syndrome; Pio, pioglitazone; Met, metformin; WT, Wortmannin; SP, SP600125; CC, Compound C.

testosterone, fasting insulin and the HOMA-IR in PCOS rats. However, in a previous study, pioglitazone monotreatment was found to markedly reduce the fasting insulin level and HOMA-IR, but to not affect the level of free and total testosterone in in women with PCOS (28). Another study showed that compared with metformin, pioglitazone treatment was less effective in improving ovulation rates, whereas the effect on hyperandrogenemia and insulin resistance was similar (37). Numerous studies have shown that metformin treatment improved the growth and development of follicles and endometrium, increased the ovulation rates and restored the menstrual cycle of PCOS patients (40-43). The present study confirmed that metformin or pioglitazone administration in a rat model of PCOS improved the follicular development, with a lower percentage of primary follicles and cystic follicles and a higher percentage of early antral follicles. Combined treatment with metformin and pioglitazone enhanced the improvement of the ovarian function of PCOS compared to that seen with the respective monotreatments. This was consistent with the results of a study using a dehydroepiandrosterone-induced PCOS rat model (33). Increasing evidence has indicated that pioglitazone and metformin improve hirsutism and insulin resistance in PCOS patients. However, the effectiveness of pioglitazone vs. metformin in the treatment of PCOS remains controversial, and the efficacy of their combined therapy in PCOS has remained to be fully demonstrated. A previous systematic review and meta-analysis of randomized controlled trials revealed that while metformin is more effective in reducing body mass index, pioglitazone is more effective in reducing insulin resistance and fasting insulin levels in women with PCOS. The effect of pioglitazone on Ferriman-Gallwey scores, testosterone levels and fasting glucose levels was not significantly different from that of metformin (44). Clomifene citrate, metformin and pioglitazone, as common insulin sensitizers, were evaluated for their effectiveness in the treatment of PCOS. There were no statistically significant differences between clomifene citrate monotreatment or its combination with metformin/pioglitazone in the biochemical pregnancy rates and the number of mature follicles (38). The efficacy of combined treatment with letrozole, metformin and pioglitazone was similar to that of combined treatment with clomifene citrate, metformin and pioglitazone within a group of 100 women with PCOS resistant to clomifene citrate (14). The present findings provided evidence that the efficacy of metformin or pioglitazone monotherapy against hyperandrogenemia, insulin resistance and follicular development was equal, whereas their combined administration showed a synergetic promoting effect. 
Furthermore, the present study provided evidence that metformin and pioglitazone ameliorate PCOS through regulating the AMPK/PI3K/JNK pathway. First, metformin and pioglitazone treatment individually or jointly inhibited JNK phosphorylation in rats with EV-induced PCOS. A similar result was found in NIT-1 cells, where metformin inhibited thapsigargin-induced JNK phosphorylation and JNK activity (34). In addition, the results of the present study showed that JNK inhibitor SP600125 significantly enhanced the combination therapy effect of metformin and pioglitazone on improving ovarian follicle development in PCOS rats. Furthermore, a previous study using SP600125 in a rat model of PCOS showed a significant improvement in ovarian follicle morphology, edema, inflammatory infiltrate, vascular congestion and hyperemia (45). These results implied that JNK activity has an important role in the pathogenesis of PCOS and that metformin and pioglitazone may regulate JNK-associated pathways. Jung et al (34) confirmed that metformin prevented endoplasmic reticulum stress-induced apoptosis through the AMPK/PI3K/JNK pathway. It is known that JNK phosphorylation is negatively regulated by PI3K/Akt and AMPK, and that PI3K has an impact on insulin signaling (46-48). PI3K inhibition is considered as a novel potential target for the treatment of PCOS (49). The present study found that using AMPK inhibitor Compound $\mathrm{C}$ and PI3K inhibitor Wortmannin significantly reduced the effect of metformin and pioglitazone combination therapy on restoring ovarian follicular development in PCOS rats. In T2DM, it has also been shown that AMPK and PI3K inhibitors inhibited metformin-mediated impairment of insulin secretion (34). Taken together, these results emphasized the significance of the AMPK/PI3K/JNK pathway in the treatment of PCOS. Metformin and pioglitazone combination therapy demonstrated great efficacy in ameliorating PCOS through regulating the AMPK/PI3K/JNK pathway.

\section{References}

1. Azziz R, Woods KS, Reyna R, Key TJ, Knochenhauer ES and Yildiz BO: The prevalence and features of the polycystic ovary syndrome in an unselected population. J Clin Endocrinol Metab 89: 2745-2749, 2004.

2. Ehrmann DA: Polycystic ovary syndrome. N Engl J Med 352: 1223-1236, 2005.

3. Teede H, Deeks A and Moran L: Polycystic ovary syndrome: A complex condition with psychological, reproductive and metabolic manifestations that impacts on health across the lifespan. BMC Med 8: 41, 2010.

4. Głuszak O, Stopińska-Głuszak U, Glinicki P Kapuścińska R, Snochowska H, Zgliczyński W and Dębski R: Phenotype and metabolic disorders in polycystic ovary syndrome. ISRN Endocrinol 2012: 569862, 2012.

5. Lee H, Oh JY, Sung YA and Chung H: Is insulin resistance an intrinsic defect in Asian polycystic ovary syndrome? Yonsei Med J 54: 609-614, 2013.

6. Dunaif A: Insulin resistance and the polycystic ovary syndrome: Mechanism and implications for pathogenesis 1. Endocr Rev 18: 774-800, 1997.

7. DeFronzo RA and Ferrannini E: Insulin resistance. A multifaceted syndrome responsible for NIDDM, obesity, hypertension, dyslipidemia, and atherosclerotic cardiovascular disease. Diabetes Care 14: 173-194, 1991.

8. Stepto NK, Cassar S, Joham AE Hutchison SK, Harrison CL, Goldstein RF and Teede HJ: Women with polycystic ovary syndrome have intrinsic insulin resistance on euglycaemichyperinsulaemic clamp. Hum Reprod 28:777-784, 2013.

9. Dehbashi S, Vafaei H, Parsanezhad MD and Alborzi S: Time of initiation of clomiphene citrate and pregnancy rate in polycystic ovarian syndrome. Int J Gynaecol Obstet 93: 44-48, 2006.
10. Palomba S, Orio F Jr, Falbo A, Manguso F, Russo T, Cascella T, Tolino A, Carmina E, Colao A and Zullo F: Prospective parallel randomized, double-blind, double-dummy controlled clinical trial comparing clomiphene citrate and metformin as the first-line treatment for ovulation induction in nonobese anovulatory women with polycystic ovary syndrome. J Clin Endocrinol Metab 90: 4068-4074, 2005.

11. Cataldo NA, Abbasi F, McLaughlin TL, Basina M, Fechner PY, Giudice LC and Reaven GM: Metabolic and ovarian effects of rosiglitazone treatment for 12 weeks in insulin-resistant women with polycystic ovary syndrome. Hum Reprod 21: 109-120, 2006.

12. Roy KK, Baruah J, Singla S, Sharma JB, Singh N, Jain SK and Goyal M: A prospective randomized trial comparing the efficacy of Letrozole and Clomiphene citrate in induction of ovulation in polycystic ovarian syndrome. J Hum Reprod Sci 5: 20-25, 2012.

13. Abu Hashim H, Shokeir T and Badawy A: Letrozole versus combined metformin and clomiphene citrate for ovulation induction in clomiphene-resistant women with polycystic ovary syndrome: A randomized controlled trial. Fertil Steril 94: 1405-1409, 2010.

14. El-khayat W, Abdel Moety G, Al Mohammady M and Hamed D: A randomized controlled trial of clomifene citrate, metformin, and pioglitazone versus letrozole, metformin, and pioglitazone for clomifene-citrate-resistant polycystic ovary syndrome. Int J Gynaecol Obstet 132: 206-209, 2016.

15. Prager R and Schernthaner G: Insulin receptor binding to monocytes, insulin secretion, and glucose tolerance following metformin treatment: Results of a double-blind cross-over study in type II diabetics. Diabetes 32: 1083-1086, 1983.

16. Goodarzi MO and Bryer-Ash M: Metformin revisited: re-evaluation of its properties and role in the pharmacopoeia of modern antidiabetic agents. Diabetes Obes Metab 7: 654-665, 2005.

17. Palomba S, Falbo A, Orio F Jr and Zullo F: Effect of preconceptional metformin on abortion risk in polycystic ovary syndrome: A systematic review and meta-analysis of randomized controlled trials. Fertil Steril 92: 1646-1658, 2009.

18. Huang X, Wang P, Tal R, Lv F, Li Y and Zhang X: A systematic review and meta-analysis of metformin among patients with polycystic ovary syndrome undergoing assisted reproductive technology procedures. Int J Gynaecol Obstet 131: 111-116, 2015.

19. Eisenhardt S, Schwarzmann N, Henschel V, Germeye A von Wolff M, Hamann A and Strowitzki T: Early effects of metformin in women with polycystic ovary syndrome: A prospective randomized, double-blind, placebo-controlled trial. J Clin Endocrinol Metab 91: 946-952, 2006.

20. Ladson G,Dodson WC, Sweet SD, Archibong AE, Kunselman AR, Demers LM, Williams NI, Coney P and Legro RS: The effects of metformin with lifestyle therapy in polycystic ovary syndrome: A randomized double-blind study. Fertil Steril 95: 1059-1066. e1-e7, 2011.

21. González-Ortiz M, Hernández-Salazar E, Kam-Ramos AM and Martínez-Abundis E: Effect of pioglitazone on insulin secretion in patients with both impaired fasting glucose and impaired glucose tolerance. Diabetes Res Clin Pract 75: 115-118, 2007.

22. Díaz-Delfín J, Morales M and Caelles C: Hypoglycemic action of thiazolidinediones/peroxisome proliferator-activated receptor gamma by inhibition of the c-Jun NH2-terminal kinase pathway. Diabetes 56: 1865-1871, 2007.

23. Xiang AH, Peters RK, Kjos SL, Marroquin A, Goico J, Ochoa C, Kawakubo $\mathrm{M}$ and Buchanan TA: Effect of pioglitazone on pancreatic beta-cell function and diabetes risk in Hispanic women with prior gestational diabetes. Diabetes 55: 517-522, 2006.

24. DeFronzo RA, Tripathy D, Schwenke DC, Banerji M, Bray GA, Buchanan TA: Clement SC, Henry RR, Hodis HN and Kitabchi AE: Pioglitazone for diabetes prevention in impaired glucose tolerance. N Engl J Med 364: 1104-1115, 2011.

25. Brettenthaler N, De Geyter C, Huber PR and Keller U: Effect of the insulin sensitizer pioglitazone on insulin resistance, hyperandrogenism, and ovulatory dysfunction in women with polycystic ovary syndrome. J Clin Endocrinol Metab 89: 3835-3840, 2004.

26. Romualdi D, Guido M, Ciampelli M, Giuliani M, Leoni F, Perri C and Lanzone A: Selective effects of pioglitazone on insulin and androgen abnormalities in normo- and hyperinsulinaemic obese patients with polycystic ovary syndrome. Hum Reprod 18: 1210-1218, 2003.

27. Lord JM, Flight IH and Norman RJ: Insulin-sensitizing drugs (metformin, troglitazone, rosiglitazone, pioglitazone, D-chiro-inositol) for polycystic ovary syndrome. Cochrane Database Syst Rev: CD003053, 2003. 
28. Stabile G, Borrielli I, Artenisio AC, Bruno LM, Benvenga S, Giunta L, La Marca A, Volpe A and Pizzo A: Effects of the insulin sensitizer pioglitazone on menstrual irregularity, insulin resistance and hyperandrogenism in young women with polycystic ovary syndrome. J Pediatr Adolesc Gynecol 27: 177-182, 2014.

29. Perez A, Zhao Z, Jacks R and Spanheimer R: Efficacy and safety of pioglitazone/metformin fixed-dose combination therapy compared with pioglitazone and metformin monotherapy in treating patients with T2DM. Curr Med Res Opin 25: 2915-2923, 2009.

30. Health NIO: Guide for the care and use of laboratory animals. National Academies, National Academy Press, 1985.

31. Mesbah F, Moslem M, Vojdani Z and Mirkhani H: Does metformin improve in vitro maturation and ultrastructure of oocytes retrieved from estradiol valerate polycystic ovary syndrome-induced rats. J Ovarian Res 8: 74, 2015.

32. Elissa LA, Elsherbiny NM and Magmomah AO: Propolis restored adiponectin level in type 2 diabetes through PPAR activation. Egyptian J Basic Applied Sci 2: 318-326, 2015

33. Di Pietro M, Parborell F, Irusta G, Pascuali N, Bas D, Bianchi MS Tesone $\mathrm{M}$ and Abramovich D: Metformin regulates ovarian angiogenesis and follicular development in a female polycystic ovary syndrome rat model. Endocrinology 156: 1453-1463, 2015.

34. Jung TW, Lee MW, Lee YJ and Kim SM: Metformin prevents endoplasmic reticulum stress-induced apoptosis through AMPK-PI3K-c-Jun NH2 pathway. Biochem Biophys Res Commun 417: 147-152, 2012.

35. Mhao NS, Al-Hilli AS, Hadi NR, Jamil DA and Al-Aubaidy HA: A comparative study to illustrate the benefits of using ethinyl estradiol-cyproterone acetate over metformin in patients with polycystic ovarian syndrome. Diabetes Metab Syndr 10 (1 Suppl 1): S95-S98, 2016

36. El Maghraby H, Nafee T, Guiziry D and Elnashar A: Randomized controlled trial of the effects of metformin versus combined oral contraceptives in adolescent PCOS women through a 24 month follow up period. Middle East Fertility Soc J 20: $131-137,2015$.

37. Ortega-González C, Luna S, Hernández L, Crespo G, Aguayo P, Arteaga-Troncoso G and Parra A: Responses of serum androgen and insulin resistance to metformin and pioglitazone in obese, insulin-resistant women with polycystic ovary syndrome. J Clin Endocrinol Metab 90: 1360-1365, 2005.

38. Abuelghar WM, Elkady OS and Khamees AA: Clomiphene citrate alone, in combination with metformin or in combination with pioglitazone as first line therapy in induction of ovulation in infertile women with polycystic ovary syndrome, a randomized controlled trial. Middle East Fertility Soc J 18: 135-141, 2013.
39. Mazza A, Fruci B, Guzzi P, D'Orrico B, Malaguarnera R, Veltri P, Fava A and Belfiore A: In PCOS patients the addition of low-dose spironolactone induces a more marked reduction of clinical and biochemical hyperandrogenism than metformin alone. Nutr Metab Cardiovasc Dis 24: 132-139, 2014.

40. Johnson N: Metformin is a reasonable first-line treatment option for non-obese women with infertility related to anovulatory polycystic ovary syndrome-a meta-analysis of randomised trials. Aust N Z J Obstet Gynaecol 51: 125-129, 2011.

41. Tang T, Lord JM, Norman RJ, Yasmin E and Balen AH Insulin-sensitising drugs (metformin, rosiglitazone, pioglitazone, D-chiro-inositol) for women with polycystic ovary syndrome, oligo amenorrhoea and subfertility. Cochrane Database Syst Rev 5: CD003053, 2012.

42. Palomba S, Falbo A and La Sala G: Effects of metformin in women with polycystic ovary syndrome treated with gonadotrophins for in vitro fertilisation and intracytoplasmic sperm injection cycles: A systematic review and meta-analysis of randomised controlled trials. BJOG 120: 267-276, 2013.

43. Palomba S, Falbo A and La Sala GB: Metformin and gonadotropins for ovulation induction in patients with polycystic ovary syndrome: A systematic review with meta-analysis of randomized controlled trials. Reprod Biol Endocrinol 12: 3 , 2014.

44. Du Q, Wang YJ, Yang S, Wu B, Han P and Zhao YY: A systematic review and meta-analysis of randomized controlled trials comparing pioglitazone versus metformin in the treatment of polycystic ovary syndrome. Curr Med Res Opin 28: 723-730, 2012.

45. Bulut G, Kurdoglu Z, Dönmez YB, Kurdoglu M and Erten R: Effects of jnk inhibitor on inflammation and fibrosis in the ovary tissue of a rat model of polycystic ovary syndrome. Int J Clin Exp Pathol 8: 8774-8785, 2015.

46. Hsu HY and Twu YC: Tumor necrosis factor-alpha-mediated protein kinases in regulation of scavenger receptor and foam cell formation on macrophage. J Biol Chem 275: 41035-41048, 2000.

47. Yin XM: Signal transduction mediated by Bid, a pro-death Bcl-2 family proteins, connects the death receptor and mitochondria apoptosis pathways. Cell Res 10: 161-167, 2000.

48. Romero L, Andrews K, Ng L, O'Rourke K, Maslen A and Kirby G: Human GSTA1-1 reduces c-Jun N-terminal kinase signalling and apoptosis in Caco-2 cells. Biochem J 400: 135-141, 2006.

49. Shah KN and Patel SS: Phosphatidylinositide 3-kinase inhibition: A new potential target for the treatment of polycystic ovarian syndrome. Pharm Bio 54: 975-983, 2016. 